\title{
ENTZUG UND BEHAUPTUNG. REAKTIONEN AUF DEN SOUVERÄNITÄTSVERLUST
}

\author{
CHRISTOPH LEPSCHY/ANDREA ZIMMERMANN
}

»Zur Demokratie gehört nicht nur die Diskussion, sondern auch die Aktion« (Rischbieter 1968: 1). So kommentierte der Theater-heute-Herausgeber Henning Rischbieter 1968 die Debatte über die Vietkong-Sammlung nach den Aufführungen des Stückes VietNam Diskurs von Peter Weiss an den Münchner Kammerspielen. Dokumentiert ist sie im Septemberheft 68 von Theater heute. Stein des Anstoßes war die Programmatik einer Aufführung, die nicht nur »)einen Missstand in der Welt bloßstellen`, sondern zur Behebung dieses Missstandes konkret auffordern will« (Schwiedrzik/Stein 1968: 3), so die Regisseure Peter Stein und Wolfgang Schwiedrzik, indem unmittelbar im Anschluss an die Vorstellung eine Sammlung für Waffen für den Vietkong durchgeführt wurde.

Knapp 40 Jahre später, im Februar 2008, resümiert der Regisseur Volker Lösch in einem Gespräch über die neuen Formen eines zeitgenössischen >politischen Theaters` eine zentrale Schwierigkeit des Theatermachens, die zugleich den Motor seines Arbeitens bildet: »Wir kommen ja gar nicht ran an die Wirklichkeit« (Behrendt/Burckhardt/Wille 2008: 12). Seine Arbeiten sind der Versuch, diesem »Erfahrungsmangel« (Merck/Pilz 2005: 17) entgegenzuwirken.

Man bemerkt den Abstand, der zwischen diesen Konzeptionen liegt: Der souveräne Gestus kritischer Selbstvergewisserung ist abhandengekommen und wird im Zeichen des Verlusts registriert. Die Tatsache, dass wir im Theater an die Wirklichkeit im Sinn einer uns unmittelbar umgebenden sozialen Realität nicht »rankommen«, hat sicher zu einem guten Teil mit den institutionellen Eigenheiten des realen Theaterbetriebs und seiner Tendenz zur Selbstreferenz zu tun. Die angesprochene Unzugänglichkeit ist aber zugleich in einem Begriff von >Wirklichkeit « selbst begründet, der totalisierenden Wahrnehmungsmustern längst nicht mehr zugänglich ist. Insofern ist der >Verlust von Wirklichkeit` als gesellschaftliches Phänomen zu begreifen, das aus der kollektiven Erfahrung der Unübersichtlichkeit und Unsicherheit einer in mehrfachen Moderni- 
sierungsschüben hoch beschleunigten Welt resultiert. Löschs Ausgangspunkt siedelt demnach im Zentrum eines Paradoxes: Aus der von sozialen Realitäten der Gesellschaft weitgehend abgekoppelten Institution Theater heraus unternimmt er Recherchen in den zersprengten, dem direkten Zugriff entzogenen Feldern unserer >Wirklichkeit<.

Die Komplexität der gesellschaftlichen Zustände lässt es nicht mehr ohne Weiteres zu, mit aufklärerischem Furor eine Position zu beziehen, von der aus wir diese nicht nur beschreiben, sondern sogar verändern könnten. Dabei besteht die Schwierigkeit vor allem darin, eine glaubwürdige Position zu beziehen, die eine kritische oder gar agitatorische Haltung überhaupt legitimiert. Wo jede Geste des Widerstands früher oder später Gefahr läuft, als Marketingstrategie verdächtigt zu werden, wo jedes Zeichen der Kritik zum Design wird, ist die Möglichkeit einer souveränen kritischen Identität nicht mehr gegeben. Aus dem Inneren eines Systems, das ebenso Anteil hat an den Ökonomisierungsprozessen wie jede andere gesellschaftliche Institution oder Firma, lassen sich schwerlich überzeugende kapitalismuskritische Positionen formulieren, ohne einer Bewegung paradoxer Selbstaufhebung anheimzufallen.

Das heißt freilich noch lange nicht, dass es falsch oder obsolet ist, solche Positionen zu suchen und zu besetzen. Das heißt auch nicht, dass es unter den Theatermachern kein Unbehagen über diese Situation gäbe, und das heißt erst recht nicht, dass die Sehnsucht nach kritischer Partizipation erloschen wäre. Im Gegenteil. Gerade das Bewusstsein dieses Souveränitätsverlustes hat in den letzten Jahren vielfache Reaktionen erzeugt und zu einer gewissen Repolitisierung der Theaterarbeit geführt, die sich dadurch auszeichnet, dass die Theater Erfahrungen außerhalb ihres angestammten Bezirks suchen und in die Theaterarbeit integrieren. Die unter diesen Prämissen entstandenen Inszenierungen können freilich nicht als gemeinsame Bewegung interpretiert werden. Umso wichtiger ist es, die einzelnen Versuche genauer anzuschauen. Im Folgenden werden wir deshalb eine Autorin und einen Regisseur und ihre Arbeitsweisen vorstellen, mit denen uns eine konkrete Arbeitserfahrung verbindet. Im Hinblick auf das formulierte Dilemma, in dem sich das Theatermachen zwischen Entzug von Wirklichkeit und der Behauptung ihrer möglichen Kritik befindet, förderte der Austausch über diese Arbeitserfahrungen überraschende Analogien in auf den ersten Blick sehr unterschiedlichen Theaterkonzepten zutage.

Nora Mansmann und Volker Lösch thematisieren den Souveränitätsverlust des Individuums und zwar vor allem, indem sie dessen Verhältnis zum Körper reflektieren. Beide zeigen das Subjekt und seinen Körper als von Machtdiskursen affiziert. Beide verfolgen dabei das Anliegen offenzulegen, dass Subjekte sowohl Medium als auch Effekt der Macht sind 
und dass Macht sozusagen >von unten` arbeitet, indem das Subjekt im dynamischen Zusammenspiel zwischen handelnden, sozialen Institutionen, kulturellen Bedeutungen, Konventionen und Beschränkungen zustande kommt. Beide insistieren dabei auf der Möglichkeit, sich zu diesem Souveränitätsverlust zu verhalten.

\section{Die Autorin Nora Mansmann}

\section{»assoziatives schreiben / ist wie gehirnjogging für mich«}

In TERRORMUM, dem ersten veröffentlichten Theaterstück von Nora Mansmann, findet sich dieses Zitat (Mansmann 2005: 23), das auch als Kommentar der 1980 in Hessen geborenen und aufgewachsenen Autorin zu ihrer Arbeitsweise gelesen werden kann: Das Schreiben von Stücken beginnt für Nora Mansmann mit dem Sammeln heterogener Textfragmente, die sie nach und nach in ein Verhältnis zueinander setzt. So entsteht aus den miteinander verwobenen Einzelteilen ein Stoff, der verschiedene Muster variiert, sodass einerseits ein struktureller Zusammenhang zwischen den einzelnen Elementen erkennbar ist, die einzelnen Motive innerhalb des Zusammenhangs andererseits ihre Selbstständigkeit bewahren. Was für die Lektüre der einzelnen Texte gilt, lässt sich für alle bisher von Nora Mansmann veröffentlichten Texte konstatieren: Bestimmte Strukturen, Themen oder Motive fallen immer wieder ins Auge. Es ist natürlich nicht auszuschließen, dass weitere Texte die Perspektive auf den Gesamtzusammenhang ihres Schreibens grundlegend verändern werden.

Die assoziative Arbeitsweise schlägt sich auch in der Mikrostruktur ihrer Texte nieder: Je nach Ort der Zäsuren, die mangels vorgegebener Interpunktion meist selbst zu setzen sind, und je nach Zuordnung variabel gehaltener Textpassagen $\mathrm{zu}$ einzelnen Figuren, verschiebt sich die Bedeutung des Textes.

Auf sehr humorvolle Weise nimmt die Autorin in ihren absurden Geschichten das Verhältnis zwischen einer Gesellschaft und der sie bildenden skurrilen Subjekte in den Blick. Spezifisch ist dabei unter anderem der von den Figuren als existenziell bedrohlich empfundene Verlust einer stabilen Identität, der in der Unfähigkeit der Figuren, souverän über ihre Körper zu verfügen, manifest wird. Das Verhältnis zwischen den Protagonisten und den Bedingungen, unter denen sie existieren, wird in verschiedenen Facetten als ein diskursiv konstruiertes ausgestellt, wie ich in 
der folgenden Lektüre der drei abendfüllenden Texte, die bisher von Nora Mansmann veröffentlicht wurden, zeigen werde.

Während in TERRORMUM (Mansmann 2005) Vater und Sohn darum ringen, ihre Subjektposition in Abhängigkeit von der abwesenden Position der Mutter zu konstruieren und aufrechtzuerhalten, stellt Nora Mansmanns zweiter Text herr tod lädt nicht ein aber wir kommen trotzdem (Mansmann 2006) die Identität der Protagonisten als medial konstruiert aus. Ihr drittes Theaterstück zwei brüder drei augen (Mansmann 2008) schließlich fokussiert auf den Körper als Gebilde, in dem sich soziale Strukturen materialisieren und das sich aufgrund permanenter Veränderung jeder Klassifikation entzieht.

\section{»anaphylaktischer schock (mein name ist nicht ulrike)«}

Bereits der Prologtitel von TERRORMUM (Mansmann 2005: 4) setzt die heftige Reaktion eines Körpers mit der einer Gesellschaft gegen die je eigene Struktur parallel: Versteht man unter einem anaphylaktischen Schock eine krankhafte Reaktion des menschlichen Immunsystems auf chemische Reize, die zum tödlichen Organversagen führen kann, ruft "mein name ist nicht ulrike« die terroristische Position von Ulrike Meinhof auf, Gründungsmitglied und intellektuelle Führungspersönlichkeit der RAF. Ähnlich wie der menschliche Körper im anaphylaktischen Schock seine Abwehrmechanismen gegen sich selbst richtet und sich zu vernichten droht, stellte der Terrorismus der RAF die bundesrepublikanische Gesellschaft mit ihren Werte- und Machtsystemen zur Disposition und zielte auf ihre Abschaffung.

In allen bisher erschienenen Texten von Nora Mansmann wird vorgeführt, wie die einzelnen Figuren von den gesellschaftlichen Verhältnissen, in denen und durch die sie existieren, durchdrungen sind. Liest man die Texte auf der Folie der Diskurstheorie nach Michel Foucault und Judith Butler, lässt sich die Wechselwirkung zwischen den Figuren und der gesellschaftlichen Wirklichkeit, in der sie leben, genauer analysieren:

Foucault geht davon aus, dass sich Diskurse, ${ }^{1}$ verstanden als sprachliche Handlungen, die die gesellschaftlichen Machtverhältnisse durchsetzen, in den einzelnen Körpern niederschlagen: »Ich suche zu zeigen, wie die Machtverhältnisse in die Tiefe der Körper materiell eindringen können, ohne von der Vorstellung der Subjekte übernommen zu werden«, so Foucault (1978: 108). Diese Machtverhältnisse, die als verschiedene

1 »Der Diskurs ist jenes regelmäßige Ensemble, das auf einer Ebene aus sprachlichen Phänomenen und auf einer anderen aus Polemik und Strategien besteht.« (Foucault 2002: 670f.) 
Diskursformationen auftreten, lassen sich nicht auf die Intention oder den freien Willen eines souveränen Subjekts zurückführen. Vielmehr setzt Foucault einem solchen transzendentalen Subjekt ein historisches Subjekt entgegen, zu dessen Konstitution zahlreiche Komponenten wie Wissen, Praktiken und Normen beitragen, die wiederum in unterschiedlichen und miteinander im Widerstreit stehenden Diskursformationen in Erscheinung treten. Diskurse bilden Wirklichkeit somit nicht ab, sondern stellen sie mittels der unzähligen Machtbeziehungen zwischen den Subjekten einer Gesellschaft her, die gleichzeitig Bedingung und Folge der in den Diskursen wirkenden Macht sind:

»Zwischen jedem Punkt eines gesellschaftlichen Körpers, zwischen einem Mann und einer Frau, in einer Familie, zwischen einem Lehrer und seinem Schüler, zwischen dem, der weiß, und dem, der nicht weiß, verlaufen Machtbeziehungen, die nicht die schlichte und einfache Projektion der großen und souveränen Macht auf die Individuen sind; sie sind eher der bewegliche und konkrete Boden, in dem die Macht sich verankert hat, die Bedingungen der Möglichkeit, damit sie funktionieren kann.« (Foucault 1978: 110)

Folglich werden die einzelnen Subjekte nicht von außen zu etwas gezwungen, sondern werden zur Selbstkontrolle angehalten, sodass Machtverhältnisse eine spezifische Form der Selbstwahrnehmung der Subjekte produzieren: »die Macht geht durch die Individuen hindurch, sie wird nicht auf sie angewandt« (Foucault 2003: 238).

Judith Butler fokussiert in ihrer Theorie, die Foucaults Diskurstheorie aufgreift und modifiziert, auf die Schaffung der Subjektposition durch Sprechakte. Sie bezieht sich dabei neben Foucault vor allem auf die Sprechakttheorie nach Austin, in der dargestellt wird, dass Sprache nicht nur einen referenziellen, sondern auch einen performativen und somit handelnden und wirklichkeitskonstituierenden Charakter hat. Auch für Butler tritt das Subjekt erst durch den Prozess der Subjektivation in Erscheinung, die im Anschluss an Foucault als Unterwerfungsprozess unter die Machtverhältnisse durch Diskurse beschrieben oder als Umwendung im Sinne von Althusser verstanden werden kann (Butler 2001: 8). Hierbei wird das Individuum in dem Moment zum Subjekt, in dem es durch einen Sprechakt angerufen wird, sich zu dieser Anrufung umwendet und sich somit der aufgerufenen Identität unterwirft. Effekt dieses Unterwerfungsprozesses ist, dass das angerufene Individuum eine Identität erhält, mit der es sozial anerkennbar ist, und so den Subjektstatus erreicht. Der Preis, den das Individuum für das Zusprechen einer handlungsfähigen Subjektposition zu zahlen hat, ist ein Normierungsprozess, denn nur die aufgerufenen Anteile des Individuums haben Platz in der intelligiblen 
Subjektformation. Alles, was nicht sozial anerkennbar ist, muss abgespalten und verworfen werden.

In TERRORMUM (Mansmann 2005) lässt sich der Prozess der Subjektkonstruktion vor allem im Verhältnis zwischen den Hauptfiguren Dennis, seinem Vater Volker und seiner Mutter beobachten. Sowohl die Position des Vaters als auch die des Sohnes ist zunächst durch die Relation zur Mutter bestimmt, obwohl diese im gesamten Stück abwesend ist. Doch der permanente Bezug auf sie und ihre politische Vergangenheit macht sie zur zentralen Figur: Das gesamte Geschehen konstruiert und verweist permanent auf die Position, die durch das Verschwinden von Dennis' Mutter zum vakanten Zentrum der Familienstruktur geworden ist.

Dennis ist »irgendwo zwischen über zwanzig und unter dreißig. versager« (Mansmann 2005: 3). An seine Mutter kann er sich nicht mehr erinnern, doch sie war - zumindest in den Erzählungen seines Vaters - eine wichtige Figur der 68er Revolution, »eine von den ganz großen« (Mansmann 2005: 3). Seit ihrem plötzlichen Verschwinden sitzt »dennis’ PAPA volker« (Mansmann 2005: 3) regungslos auf dem Sofa und lebt nun seit Jahren beinahe ausschließlich in der verklärenden Erinnerung an diese große Zeit. Ohne der politischen Bedeutsamkeit seiner Mutter etwas Eigenes entgegensetzen zu können, beschließt Dennis, basierend auf den Erzählungen seines Vaters, ein Buch über ihre Geschichte zu schreiben: Projekt TERRORMUM. So wird die verschwundene Mutter durch permanentes Zitieren zum zentralen - und doch eigentlich abwesenden Referenzpunkt für die Subjektpositionen von Mann und Sohn, dessen Aufrechterhaltung zunächst für beide überlebenswichtig ist. Während Dennis versucht, auf der Vergangenheit seiner Mutter seine eigene $\mathrm{Zu}$ kunft aufzubauen, hat sich der Vater entschieden, jenseits eines Sprechens, das fast ausschließlich im Zitieren dieser Vergangenheit besteht, seinen Körper stillzulegen. Er verharrt in einer Art »ohnmacht« oder »stand-by-zustand" (Mansmann 2005: 4): »jetzt hab ich die bedienungsanleitung für mich vergessen hab sie nicht dabei wie blöd« (Mansmann 2005: 4). Verbunden mit dieser Konzentration auf die Vergangenheit ist so die Weigerung, sich zur Gegenwart zu verhalten. Er antwortet ausschließlich auf die Anrufung als Mann der großen Revolutionärin und wahrt dadurch seine Subjektposition. Lediglich das Erzählen seiner Biografie, die sich für ihn ausschließlich in Relation zu »MAMA« (Mansmann 2005: 3) bestimmt, trennt ihn von der gesellschaftlichen NichtExistenz. $\mathrm{Ob}$ er jenseits dieser permanenten Rekonstruktion existieren kann, ist fraglich. Als Dennis das Projekt TERRORMUM abbricht, um einen Science-Fiction-Roman zu schreiben, antwortet »PAPA« gar nicht mehr, entzieht sich endgültig dem Diskurs und gibt damit seine Existenz 
als wahrnehmbares und handlungsfähiges Subjekt auf. Für Dennis hingegen bedeutet die Aufgabe des Projekts TERRORMUM, sich aus der alles beherrschenden Vergangenheit zu lösen, seine Gegenwart als Subjekt zu gestalten, seinen Ort in der ständig performativ wiederhergestellten Familienkonstruktion zu verlassen und sich schreibend eine mögliche $\mathrm{Zu}$ kunft und mögliche Welten zu erschließen.

\section{»einmal im leben richtig spaß haben oder bonny und clyde «}

Auch in ihrem zweiten Stück herr tod lädt nicht ein aber wir kommen trotzdem (Mansmann 2006) nimmt Nora Mansmann die Kinder der 68er in den Blick, beschreibt in drei assoziativ verbundenen Variationen, wie im Prologtitel bereits angedeutet (Mansmann 2006: 4), jedoch ein Lebensgefühl, das sich an einer medial vermittelten Wirklichkeit orientiert. Hilflos versuchen die Figuren, sich zu den Protagonisten ihres eigenen Films zu machen, dadurch Originalität zu erlangen und der tödlichen Langeweile ihres Alltags zu entkommen. Orientierungslos und zwischen Resignation und Aufbruch (fragt sich nur wohin) probieren die Figuren verschiedene Leitbilder aus, die sie von der Leinwand kennen. Hollywood-Helden und Terroristen werden Vorlage einer ziellosen Nachahmung. Wissen die beiden Protagonisten auf der Suche nach dem »roadmovie-gefühl« (Mansmann 2006: 5) nicht mehr, wie das Spiel der Geiselnahme weitergeht, steigen sie einfach aus ihrer Rolle aus. Das Spiel mit dem Tod - ob mit dem eigenen oder dem eines anderen Menschen scheint die einzige Möglichkeit, zumindest über den Gedanken der Endlichkeit kurzfristig ein Gefühl für die eigene Bedeutung zu erlangen. Sie flirten mit dem Tod und spielen wie mit der Fernbedienung in der Hand die verschiedenen Szenarien des Sterbens durch, denn »je näher du dem tod kommst desto lebendiger fühlst du dich« (Mansmann 2006: 29).

Judith Butler hat gezeigt, wie die performativ hergestellten Normen der herrschenden Diskurse ihre Herstellungsprozesse verschleiern und sich den Anschein des Ursprünglichen oder Natürlichen geben. Hollywood-Helden sind solche inkorporierte Normen, die als Originale wahrgenommen und medial vermittelt werden. Angehalten zur Selbstkontrolle versuchen die Subjekte einer Gesellschaft, diesen Normen in Bezug auf Lebensstil und Körperpraxis zu entsprechen. herr tod lädt nicht ein aber wir kommen trotzdem führt somit die paradoxe Situation zweier Subjekte vor Augen, die im Versuch, selbst zum Original zu werden, die Normen zitieren und performativ aufrechterhalten. 


\section{»heute morgen bin ich aufgewacht und mein geschlecht war weg«}

Auf den ersten Blick sind vor allem die Körper der Figuren in Nora Mansmanns drittem Stück zwei brüder drei augen (Mansmann 2008) den sich ständig wandelnden Bedingungen ihrer Umwelt ausgeliefert: Alle Figuren sind permanent von spontanen Mutationen bedroht, nur »OMA« (Mansmann 2008: 3) liegt in ihrem Bett fixiert im Teilzeitkoma und stellt gleichsam einen Fixpunkt im Zentrum der Geschehnisse dar. In ihrer Wohnung leben auch ihre beiden Enkel: »FROTZI hermaphroditIn« und »WOWA ihr verblödeter bruder, dreiäugig« (Mansmann 2008: 3). Während sie für Frotzi nur noch als bedauernswerter Körper anwesend ist "unsere oma wirklich traurig zu sehen was mal aus uns werden wird da liegt man dann als hirnlose masse und niemand stellt die apparate ab« (Mansmann 2008: 11) -, unterhält Wowa weiterhin eine Beziehung zu ihr, spricht sie als Subjekt an und erhält auch Antwort.

»omas wohnung« (Mansmann 2008: 6) ist der letzte Ort, an der sich die Behauptung einer bürgerlichen Ordnung zunächst noch aufrecht erhalten lässt, und wird im Verlauf des Stückes immer mehr zur (scheinbar möglichen) Zuflucht vor einer Wirklichkeit, die in psychologischer, politischer und physikalischer Hinsicht alle bisher angenommenen Gesetzmäßigkeiten überschreitet. Dies betrifft auch die Figuren, die bereits im Prolog als Mutanten charakterisiert werden. Sie können sich nicht mehr auf kontinuierlich definierte Körpergrenzen beziehen, wie sie zur Beschreibung einer intelligiblen Identität notwendig sind. Umso wichtiger ist für sie das Sprechen als Akt der Konstitution und Vergewisserung als Subjekte:

»der urknall damit fängt es an wir sprechen

ein universum explodiert im kopf

aber was soll das hier

dieser urschleim oder was das ist

dieser schleimige urschleim aus dem wir bestehen entstehen irgendwas organisches zellen haufen haufen von zellen körper formen konturen augen schwarz ohren schwarz hände haut gesicht körper schwarz alles immer schwarz immer noch aber wir sind da wir sprechen wir sind der raum scheiß dunkler riesen raum ohne decke ohne boden ohne wände ohne aua wir wissen gar nicht wo genau wir sind und was es gibt keine orientierung es gibt nichts zu essen vielleicht gibt es wenigstens ein sofa« (Mansmann 2008: 4).

Durch Sprache entstehen aus einer Anhäufung von Zellen die einzelnen Figuren, werden die Individuen zu einzelnen erkennbaren Subjekten und 
somit erst handlungsfähig. Der Prolog lässt sich also parallel zu dem von Butler beschriebenen Prozess der Subjektivation lesen:

»Individuen besetzen die Stelle, den Ort des Subjekts (als welcher `Ort` das Subjekt zugleich entsteht), und verständlich werden sie nur, soweit sie gleichsam zunächst in die Sprache eingeführt werden. Das Subjekt ist die sprachliche Gelegenheit des Individuums, Verständlichkeit zu gewinnen und zu reproduzieren, also die sprachliche Bedingung seiner Existenz und Handlungsfähigkeit.« (Butler 2001: 15f.)

Der Prozess der Subjektwerdung ist jedoch nie abgeschlossen. Die mit der Subjektposition geforderte Matrix der Intelligibilität muss durch einzelne Akte immer wieder zitathaft aufgerufen und bestätigt werden. Butler greift mit einer solchen performativen Herstellung der Identität einen Gedanken von Jacques Derrida auf, der im Anschluss an die Zeichentheorie de Saussures und deren Weiterentwicklung durch Lacan die arbiträre und differenzielle Konstitution der Signifikate durch die Signifikanten betont. Bedeutung entsteht in diesem Sinne erst durch die dynamische Strukturation der Zeichen untereinander: Sowohl Wiedererkennbarkeit verbunden mit zwangsläufigen Vorannahmen als auch permanente Bedeutungsverschiebung durch die jeweilige Aktualisierung und Kontextualisierung der Zeichen sind für Sprache konstitutiv (Derrida 1976). Für Normen und gesellschaftlich intelligible Subjektpositionen bedeutet dies, dass sie mittels Zitieren einerseits kontinuierlich hergestellt werden, die einzelnen Akte ihrer Aktualisierung andererseits individuelle Bedeutungen und somit Bedeutungsverschiebungen produzieren. ${ }^{2}$

Vor allem in Bezug auf die Hauptfiguren von zwei brüder drei augen lässt sich beobachten, wie sie dadurch in eine zunehmend prekäre Lage geraten, dass ihnen konventionelle Differenzen als Referenzpunkte, die bisher eine verlässliche Deutung ihrer Wirklichkeit möglich machten, in wachsender Geschwindigkeit abhandenkommen: Wowa hat durch sein drittes Auge beängstigende apokalyptische Visionen, in denen sich die Differenz zwischen Organischem und Anorganischem auflöst: Treppenhäuser, Fahrstühle und Zimmer werden zu Teilen eines riesigen lebenden Organismus. Diese Visionen, die als Blick in die Zukunft auch die Differenz zwischen den Zeitdimensionen auflösen, hindern ihn daran, die scheinbar sichere Wohnung zu verlassen; doch auch die Unterscheidung von Innen- und Außenraum wird zunehmend schwieriger. Zuflucht

2 »Die Kraft und die Bedeutung einer Äußerung sind nicht ausschließlich durch frühere Kontexte oder >Positionen` determiniert; eine Äußerung kann ihre Kraft gerade aus dem Bruch mit dem Kontext gewinnen, den sie ausführt« (Butler 2006: 227). 
nimmt Wowa zum einen immer wieder in der selbstvergewissernden sprachlichen Beschreibung der Situationen. Zum anderen fahndet er permanent nach der Antwort auf die Frage: »was ist schönheit? (Mansmann 2008: 4), in der Hoffnung, dass diese ihm, einer Zauberformel gleich, (ästhetische) Kategorien zugänglich machen könnte, die ihm seine Wirklichkeit in einem sinnvollen Zusammenhang zu erschließen vermögen.

Besonders prekär ist die Situation für Frotzi, die sich jenseits der für eine intelligible Subjektposition konstitutiven Matrix der Geschlechtsidentität aufhält. Judith Butler stellt die heterosexuelle Matrix, die bestimmt, wie sich biologisches Geschlecht, kulturelles Geschlecht und Begehren im Sinne einer klar konturierten Identität zueinander zu verhalten haben, als grundlegende Bedingung für die Intelligibilität des Subjekts heraus. Wer sich jenseits dieser Matrix aufhält, kann in der Gesellschaft keine Position als anerkennbares und handlungsfähiges Subjekt einnehmen. Bei Mansmann heißt es:

»heute morgen bin ich aufgewacht und mein geschlecht war weg

ja das passiert mir in letzter zeit öfter

zuerst war das nur für kurze zeit und dann war es plötzlich wieder da (...)

und es ist nicht nur das geschlecht das kam zuerst

aber jetzt der körper der ganze körper ich verliere meinen körper er löst sich auf und ich sitz da und guck von irgendwo ich weiß nicht wo

von außen?

auf mich drauf und finde alles so absurd dass die so mit mir reden als wäre nichts passiert als

wär ich ganz normal als wär ich wirklich da

bin aber nicht im körper sondern außerhalb

und gleichzeitig innerhalb

bin irgendwie überall

die grenzen verschwimmen die grenzen nach außen

und dieses ding da im spiegel soll ich sein da muss ich lachen

mein ich was ist das denn

wo ist das denn

was ist denn das

ich weiß manchmal gar nicht ob es mich eigentlich noch gibt«

(Mansmann 2008: 33).

Die Dekonstruktion des zunächst als weiblich anerkennbar konstruierten Körpers wird zur existenziellen Not. Als »hermaphroditIn« kann sich Frotzi im binären Zwei-Geschlechter-Modell nicht mehr verorten. Als sie sich in Nobbi, einen »freundlichen, älteren werwolf« (Mansmann 2008: 3), verliebt, wird die Situation nicht gerade einfacher: Es ist Frotzi unmöglich, die eigene (Geschlechts-)Identität als verlässliche und konti- 
nuierliche Position zu erfahren, und die gerade beginnende Beziehung zu Nobbi trägt durch die nicht einzuordnende und instabile Begehrensstruktur zum Chaos in der Geschlechtermatrix bei.

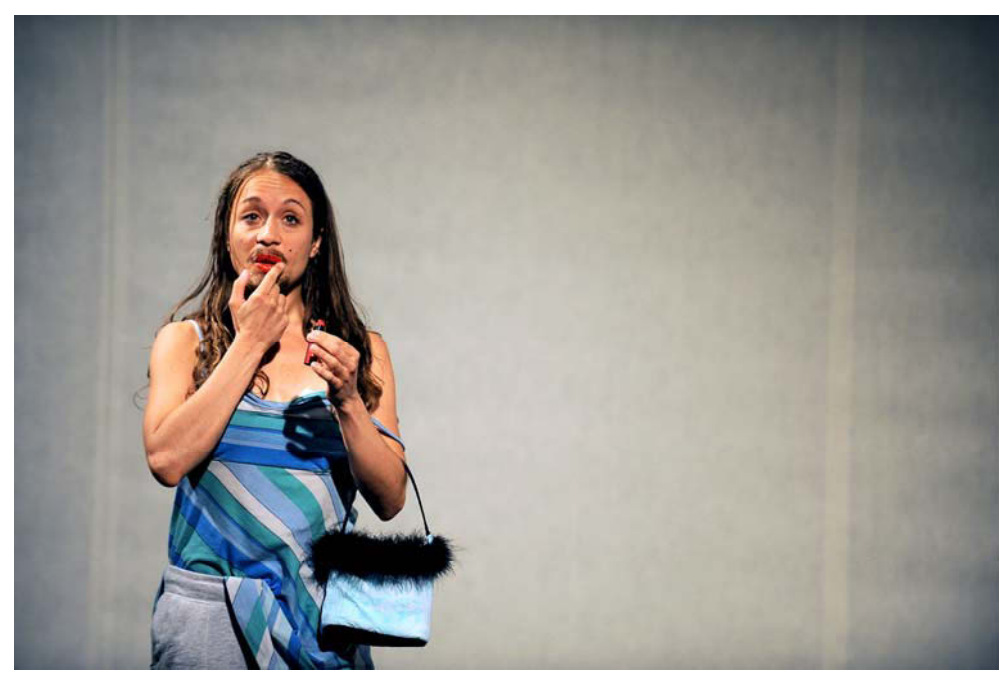

Abbildung 6: Janina Sachau als Frotzi

in der Uraufführung von zwei brüder drei augen,

20. Juni 2008, Düsseldorfer Schauspielhaus, Regie: Christian Doll (Foto: Sebastian Hoppe)

Dabei ist auch Nobbi permanenten Veränderungen ausgesetzt: Abgesehen von spontanen Mutationen zum Werwolf, an die er sich bereits gewöhnt hat, entpuppt sich eine Beule an seinem Kopf als singender Tumor namens »STALIN" (Mansmann 2008: 3), der ihn nach und nach >übernimmt . Er kann keinen Bezug zu sich selbst mehr herstellen, da seine Identität unterlaufen wird. Das Ich scheint auseinanderzufallen oder sich immer weiter auszudehnen. So sind auch seine Körpergrenzen nicht mehr wahrnehmbar. Das Selbst, das im Spiegel reflektiert wird, kann nicht mehr in Bezug zu einem Ich gesetzt werden:

»es ist kein schönes gefühl im moment erscheint es mir als würde ich einfach immer dicker werden aufgeblasen werden oder aufgeschwemmt ich hab nichts gemacht nichts geändert weiß nicht wo das herkommt weiß nicht wo das hinführt kann mich selbst schon fast nicht mehr erkennen im spiegel oder mein spiegelbild in der fensterscheibe« (Mansmann 2008: 4). 
Frotzi und Nobbi suchen in der Beziehung zueinander nach gegenseitiger Vergewisserung ihrer Identität und deren Bedeutung. Doch diese Sehnsucht erfüllt sich nicht:

/scheiß mutationen mir reichts

nobbi kommt vorbei

wir haben sex

und seine äußere körperform ändert sich ständig« (Mansmann 2008: 31).

Zum >Glück ' haben alle eine Therapeutin: »DR. FOXY, hno-ärztin und psychotherapeutin« (Mansmann 2008: 3). Pillen, Tabletten und Therapien sollen die Probleme in den Griff bekommen. Doch Dr. Foxy nutzt die Sitzungen lediglich zum Sammeln wichtiger Informationen, schließlich will sie mithilfe des »GROSSEN TUMORS« (Mansmann 2008: 17) die Weltherrschaft übernehmen. Therapie als Mittel der Selbstreflexion wird ad absurdum geführt, ist vielmehr Einfallstor für Manipulation durch die Therapeutin und den Diskurs der Medizin, dessen Autorität als zugeschrieben entlarvt wird. Der Versuch, eine natürlich erscheinende (Geschlechts-)Identität durch Medikation und Therapie zu produzieren, wird ausgestellt, die Matrix der Intelligibilität wird des Anscheins der Natürlichkeit beraubt und die Kontingenz ihrer Normen offensichtlich.

So steuert alles auf den Weltuntergang $\mathrm{zu}$, als mit der Ununterscheidbarkeit zwischen Ich und Anderem die letzte Differenz fällt. Die Welt mutiert und mit ihr alle Lebewesen. Das Ziel von DR. FOXY scheint erreicht:

»die reine harmonie die reine utopie das ist es das ist unser ziel ihr wisst nicht was gut für euch ist wir werden alle eins sein zusammenmutieren metastasieren alle zusammen unter der obhut des GROSSEN TUMORS« (Mansmann 2008: 42).

\section{Der Regisseur Volker Lösch}

Auf den ersten Blick haben der Regisseur Volker Lösch und die Autorin Nora Mansmann wenig miteinander zu tun. Während bei Mansmann die Realitätsaneignung in filigran gearbeiteten, assoziativen Textnetzen verläuft, arbeitet Volker Lösch in seinen Inszenierungen mit heterogenem Material aus biografischen Erfahrungen, Texten und Personen aus der >unmittelbaren Wirklichkeit<. Was sie verbindet, ist der Versuch, mit unterschiedlichen Mitteln auf das gesellschaftliche Bewusstsein des Souveränitätsverlustes zu reagieren statt es nur zu beschreiben. 
Volker Löschs Theater verwendet eine Reihe von Strategien zur Wirklichkeitsaneignung mit dem Ziel, die Grenzen zwischen Theater und gesellschaftlicher Realität durchlässiger zu machen. Diese sollen hier strukturell beschrieben und anhand eines Beispiels aus der Arbeitspraxis plastisch werden.

\section{Geografische Verortung}

Der gemeinsame Ausgangspunkt oder vielleicht besser: Angriffspunkt fast aller Inszenierungen von Lösch ist zunächst eine ganz konkrete geografische Verortung. Lösch macht Theater ausgehend von dem Ort, an dem er jeweils arbeitet. Er findet die Bezugspunkte für seine Arbeiten in der urbanen Gesellschaft in der unmittelbaren Umgebung der Theater, an denen er inszeniert. $\mathrm{Ob}$ es die Migrantinnen in der Stuttgarter Medea ${ }^{3}$ (die einen realen Raum konkreter Fremdheitserfahrung öffnen), ob es der Rechtsextremismus im Dresdner Woyzeck ${ }^{4}$ (mit dem Dresdner Bürgerchor, der Texte aus einer Umfrage unter Dresdner Bürgern präsentierte) oder die biografischen Erfahrungen von Privatbankrotteuren in der Düsseldorfer Inszenierung Besuch der alten Dame ${ }^{5}$ sind: Stets wird der geografische Ort auch als sozialer Ort begriffen. Lösch und sein Team recherchieren, manchmal gemeinsam mit den Schauspielern, an diesen Orten offener oder versteckter gesellschaftlicher Auseinandersetzungen und er implementiert die vorgefundenen Diskurse direkt in seine Aufführungen.

Man könnte sagen, wo bei Nora Mansmann der Bezug auf den deformierten Individualkörper den Ausgangspunkt bildet, ist es bei Lösch das konkrete Segment eines gesellschaftlichen Körpers, das er auf die Bühne zu stellen versucht. Das geschieht einerseits über die Montage von dokumentarischem Textmaterial, andererseits über die Integration von Laien (den derzeit populärsten Repräsentanten der >Wirklichkeit` auf deutschen Bühnen) in das Bühnengeschehen, vor allem in Form des Chores.

\section{Zitat und Montage}

Die Inszenierungen von Volker Lösch bewegen sich dabei oft in einem Raster von Figur und Handlung, das ganz in der Tradition eines klassi-

3 Staatsschauspiel Stuttgart, Premiere am 26.05.2007

4 Staatsschauspiel Dresden, Premiere am 12.10.2007.

5 Düsseldorfer Schauspielhaus, Premiere am 09.02.2007. 
schen Dramenbegriffs steht. Allerdings bringt er ihn in Spannung mit einer Form von kollektiver Autorschaft, die mittlerweile alle seine Projekte auszeichnet. Häufig greift er dafür auf bewährte Texte aus dem Schauspielrepertoire (Medea, Woyzeck, Die Weber, Der Revisor, Der Besuch der alten Dame, Hamlet et cetera) zurück, die er gleichsam als Basisgeschichten nutzt und im Lauf des Arbeitsprozesses mit dokumentarischen Texten durchsetzt und anreichert. Diese stammen aus Interviews, aus der Presse, aus dem Internet, es sind Reden von Politikern, Wirtschaftsvertretern und anderen Prominenten, es sind Presseerklärungen oder biografische Erzählungen.

Der allusive Umgang mit Theatertexten, der auf den identifikatorischen Wert vieler klassischer Texte setzt, und den herauszuarbeiten nach wie vor als ein herrschendes Paradigma der Theaterpraxis gelten kann, ist nicht sein Arbeitsfeld. Lösch interessiert sich nicht dafür, die sogenannte Aktualität im überlieferten Text behutsam freizulegen und assoziativ Gegenwartsbezüge zu erzeugen. Ihm liegt daran, die Stücke unmittelbar mit dem Blick auf konkrete gesellschaftliche Verwerfungen zu erzählen. Die dafür benötigten Fremdtexte werden häufig im Rahmen von ausgreifenden Recherchen in denjenigen gesellschaftlichen Segmenten gewonnen, die später im Zentrum der jeweiligen Inszenierung stehen. Sie erscheinen dann entweder als unmittelbare Figurenreden und treten an die Stelle der ursprünglichen Dialoge oder sie werden zu Sprachpartituren der Chöre verarbeitet.

In der erwähnten Stuttgarter Medea wurde ein Chor aus 16 türkischen Migrantinnen, die in Interviews von ihren Fremdheitserfahrungen in der schwäbischen Metropole erzählten, in die Tragödie des Euripides eingefügt. Für den Dresdner Woyzeck wurden Interviews mit 529 Dresdner Bürgern über ihre Haltung zu Rechtsextremismus und Gewalt geführt. In beiden Fällen wurden diese individuellen Berichte zu Chortexten kompiliert und ersetzten oder ergänzten die ursprünglichen Dialoge des Stücks. Die subjektiven Erfahrungen werden dabei im Chor entpersonalisiert und unter dramaturgischen Gesichtspunkten montiert. In $\mathrm{Me}$ dea sind es die Migrantinnen selbst, die auf der Bühne stehen, in Dresden ist es ein Bürgerchor, der sich im Laufe von insgesamt drei Projekten (Orestie, Die Weber, Woyzeck) gebildet hat. Entscheidend ist, dass das Material zwar von Laiendarstellern, die den thematisierten sozialen Milieus entstammen, präsentiert wird, die chorische Form jedoch jeden Anspruch auf unmittelbare Authentizität dementiert. Man könnte also sagen, dass dieses Prinzip stets den Widerspruch zwischen jener vermeintlichen Authentizität, die die individuellen Darsteller garantieren, und einer Darstellungsform, die deren persönliche Erzählungen im Chor anonymisiert, offenhält und austrägt. 
Ähnliches gilt allerdings auch meist für die individuelle Sprache der Einzelfiguren in diesen Projekten: Der zitathafte Charakter des Sprechens wird keineswegs verschleiert und tritt umso deutlicher hervor, je erkennbarer die diskursiven Bruchstücke zwischen den Originaltexten bleiben und sich mit diesen wechselseitig ergänzen. Indem dieses Sprechen auf seine diskursive Herkunft verweist und dabei manchmal geradezu schablonenhaft erscheint, dementiert es implizit ebenfalls den Gestus authentischer Figurenrede.

Auf der literarischen Ebene ist das Resultat dementsprechend von großer Heterogenität, es sind Montagen unterschiedlicher Textsorten disparater Qualitäten, die nicht mehr oder kaum von einem einzelnen Autorenwillen durchformt sind und ihren zitathaften Kompilationscharakter nicht verbergen. Auf der Ebene der Darstellung aber entsteht die Kraft eines Kollektivs, das den formulierten Problemen biografisch verbunden ist und sie entsprechend zu beglaubigen vermag.

\section{Der Besuch der alten Dame}

Der Prozess einer kollektiven Texterfindung war auch die Basis für die Arbeit an Der Besuch der alten Dame von Friedrich Dürrenmatt am Düsseldorfer Schauspielhaus, eine Arbeit, die zwar ohne Chor auskommt, dafür aber die Schauspieler als Autorenkollektiv an der Neufassung des Stücks beteiligte.

Das Stück ist ein gutes Beispiel für einen Text, dessen allgemein verbindliche Kapitalismuskritik mittlerweile jegliche Schärfe verloren hat und mit dessen Grundaussage sich jedermann ohne Schwierigkeit identifizieren wird. Dafür sorgt schon die durch die Gattung der Parabel bedingte und in der Wahl des Ortes hervorgehobene Allgemeingültigkeit: Güllen, so Dürrenmatt, ist eine Kleinstadt »irgendwo in Mitteleuropa« (Dürrenmatt 1998: 141), die Zeit, ganz lapidar, die »Gegenwart« (Dürrenmatt 1998: 12). Dabei steckt in der dramaturgischen Struktur des Stückes nach wie vor erhebliches polemisches Potenzial, vor allem im Hinblick auf die Frage nach der Definitionsmacht des Geldes über den Begriff und die Praxis von >Gerechtigkeit beziehungsweise auf das komplizierte Abhängigkeitsgefüge von Schuld und Schulden.

Im Sinne der oben skizzierten Arbeitsweise galt es nun, Dürrenmatts Forderung nach Gegenwart wörtlich zu nehmen und das Stück dafür zunächst geografisch zu verorten. Für ein Stück über die Macht des Geldes erwies sich Düsseldorf als der geradezu klischeehaft adäquate Ort. Aus dieser Setzung ergaben sich Konsequenzen: Das Stück spielt im Original in einer verfallenden Kleinstadt mit verarmtem Gemeinwesen. Der Hand- 
lungsort aber sollte jetzt eine wohlhabende Großstadt sein. Damit stand auch die Grundvoraussetzung der Handlungslogik des Stückes, die Not und die Erpressbarkeit der Stadtbevölkerung ebenso wie die Charakterzeichnung und Konstellation der einzelnen Figuren zur Disposition. In Dürrenmatts Stück entfaltet das unmoralische Angebot der alten Dame, um den Preis des Todes eines Mitbürgers einer ganzen Stadt zu Wohlstand zu verhelfen, seine Wirkung vor dem Hintergrund einer verarmten (Nachkriegs-)Gesellschaft. Gegenwärtig liefern die allgegenwärtigen Verlustängste längst ein gesellschaftliches Panorama, das sich als Experimentierfeld für Claire Zachanassian ebenso tauglich erweist. Die Erhaltung des Wohlstands als ultimative Handlungsmaxime sorgt für ausreichende Erpressbarkeit und bietet einen wirksamen Ansatzpunkt für die Versuchsanordnung Dürrenmatts.

Wir begannen uns mit dem Reichtum in Düsseldorf zu beschäftigen und stießen rasch auf das Thema Schulden: Düsseldorf ist zwar als Stadt schuldenfrei (und damit strukturell weit entfernt von Dürrenmatts imaginärem Güllen), aber die Quote der Privatverschuldung, das heißt die Quote der in Düsseldorf lebenden privaten Bankrotteure, liegt deutlich über dem Landes- und Bundesdurchschnitt. Recherchen führten uns $\mathrm{zu}$ Schuldnerberatungen, kleinen und großen Schuldnern, wir sammelten Geschichten über Menschen, die auf unterschiedliche Weise über ihre Verhältnisse leben, erfuhren von ihren Strategien damit zu leben, von den Augenblicken des Bankrotts und gewannen mehr und mehr Material für die Biografien der Figuren. Schließlich ließ sich die Setzung Dürrenmatts von der existenziellen Not seiner Stadtbewohner unter den Bedingungen der zeitgenössischen Schuldenfalle neu perspektivieren und als Kampf um den Statuserhalt des Einzelnen in einer wohlhabenden Gesellschaft weiterverfolgen.

Das Motiv der verschuldeten Gesellschaft ist auch im zweiten Teil von Dürrenmatts Originaltext ein entscheidender Hebel, wenn sich die Güllener maßlos und mutwillig verschulden (indem sie unter anderem Vollmilch und Cognac kaufen) und damit selbst jenen Druck erzeugen, dem sie schließlich Alfred Ill opfern. Dementsprechend galt es einen für unsere Version adäquaten Verschuldungsprozess zu erfinden, der einen Fluchtpunkt für die mit dem Geld der Claire Zachanassian verbundenen Erlösungshoffnungen zu öffnen vermag.

Wie oben im Abschnitt über Nora Mansmann ausgeführt, schlagen die gesellschaftlichen Zusammenhänge stets auf die Formierung und Deformierung der Körper zurück. Wenn bei Mansmann Körper als Gebilde erscheinen, in denen sich explizit soziale Strukturen manifestieren, lag es im Fall von Löschs Güllener Düsseldorfern nahe, den umgekehrten Weg 


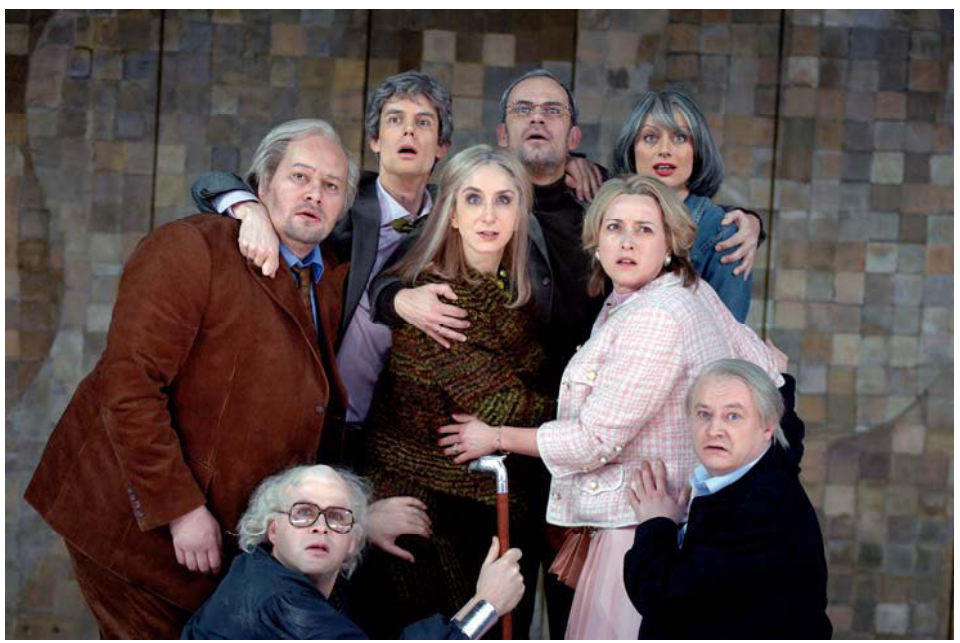

Abbildung 7: Düsseldorfer Schauspielhaus, Der Besuch der alten Dame, Rainer Galke, Hans-Jochen Wagner, Urs Peter Halter,

Claudia Hübbecker, Matthias Leja, Katharina Abt, Cathleen Baumann, Christoph Müller

(Foto: Sebastian Hoppe)

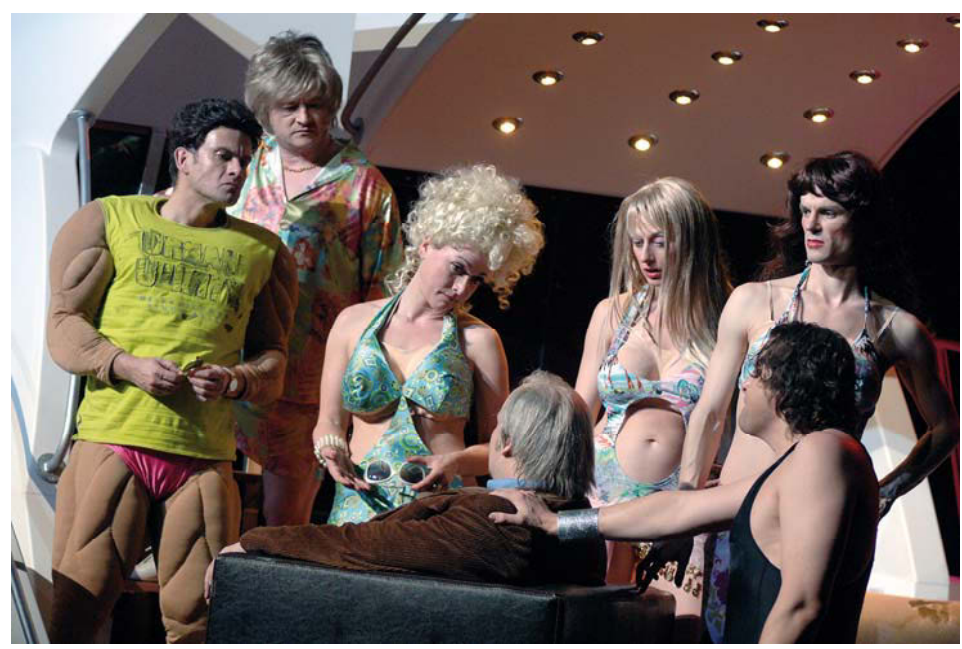

Abbildung 8: Düsseldorfer Schauspielhaus, Der Besuch der alten Dame, Matthias Leja, Christoph Müller, Katharina Abt, Rainer Galke, Cathleen Baumann, Urs Peter Halter, Hans-Jochen Wagner (Foto: Sebastian Hoppe) 
einzuschlagen und den plastischen Chirurgen aufzusuchen: Denn die kosmetische Chirurgie suggeriert, Identitäten modellieren zu können, sie entzaubert den Mythos der ewigen Jugend und rückt ihn in die Sphäre ökonomischer Verfügbarkeit. Mit der Verfügbarkeit der Körper aber verbindet sich die Hoffnung auf Glück. Dergestalt erscheint am Horizont der kollektiven Erlösung die chirurgische Modellierung der Identität im Zeichen ewiger Jugend. Für die Inszenierung hieß das: Die maßgeblichen Güllener sind zu Beginn alle um die 60 und werden im Laufe des zweiten Akts immer jünger - außer Alfred Ill.

Von diesen vor Probenbeginn vorgenommenen Setzungen - Großstadtbewohner, Privatbankrotteure, Patienten der plastischen Chirurgie - ausgehend, erweiterten wir nun das ursprüngliche Personal und erfanden neue Figuren hinzu (zum Beispiel Medienmanager und Kunstprofessor). Die Recherchen der Dramaturgie wurden gemeinsam mit den Schauspielern fortgesetzt. In Improvisationen mit den gesammelten und erfundenen Texten, häufig auf der Grundlage von Dürrenmatts Dialogen, dabei fast immer seinem dramaturgischen Gerüst folgend, entstand eine zeitgenössische Variation des Stücks. Dabei ist der zitathafte Charakter der Sprache manchmal offensichtlich, manchmal verbinden sich Zitat und Erfindung mit Dürrenmatts Original zu eigenwertigen Dialogpassagen. Immer offen bleibt so allerdings die Frage nach der Herkunft eines Wortes, eines Satzes. Das offensichtliche Verfahren der Textverfremdung macht es unmöglich, die einzelnen Äußerungen ausschließlich als gesicherte und psychologisch begründbare Figurenreden zu interpretieren. Ständig weisen diese Reden über sich hinaus und reflektieren ihr eigenes Zustandekommen.

Ein wichtiger Aspekt ist in diesem Zusammenhang auch das ironische Spiel der Inszenierung mit fadenscheinigen Gesten der Publikumspartizipation: Die Schauspieler sitzen anfangs im Publikum, das Publikum wird zum Mitsingen aufgefordert. Der karnevalistische Vergnügungsgestus erzeugt eine größtmögliche Identifikation des Publikums. Am Schluss werden die Zuschauer zur Abstimmung über den Tod von Alfred Ill gebeten und auf perfide Weise für seine Ermordung mitverantwortlich gemacht: »Wer sitzen bleibt, ist dafür.« Der Gestus vermeintlicher Publikumspartizipation funktioniert auch hier reibungslos und reflektiert ziemlich genau ein zeitgenössisches kollektives Ohnmachtsgefühl. Das Spiel mit der Theaterkonvention macht auf eine Struktur aufmerksam, die so selbstverständlich und geläufig geworden ist, dass sie unterhalb der Wahrnehmungsschwelle liegt. Lösch operiert hier auf ähnliche Weise wie bei der Sprachbehandlung der Figuren: Indem er eine Konvention verwendet und sie zugleich ausstellt, wird deren »Verwen- 
dung zu einem expliziten Beispiel von Diskursivität [...], das eher reflektiert als für ein gesichertes Verfahren der Normalsprache gehalten werden sollte.« (Butler 2006: 158) An diesem Punkt berühren sich die poetischen Verfahren von Nora Mansmann und Volker Lösch fast explizit. Auch im Schlussbild ist dieses >diskursive Spiel« wirksam: Die Hinrichtung von Alfred Ill ist als religiöses Opferritual inszeniert, das von den Güllenern in einem Chorsatz gerechtfertigt wird, der aus Versatzstücken der allgegenwärtigen Opferrhetorik in politischen und ökonomischen Diskursen besteht und so auf deren religiöse Dimension aufmerksam macht.

\section{Fazit. \\ »ist das nicht schön verschwinden und gleichzeitig eins sein mit allem «}

Nora Mansmann zeichnet mit den Mitteln der Groteske und der Ironie das Bild einer postideologischen Generation, die zum einen mit den Aufräumarbeiten der Mythen ihrer Vorgänger beschäftigt ist und zum anderen permanenten Verschiebungen innerhalb der gesellschaftlichen Machtverhältnisse ausgesetzt ist, die weder überblickt noch gesteuert werden können. Wie im Schlusszitat von zwei brüder drei augen (Mansmann 2008: 47) werden in ihren Stücken die Konsequenzen der diskursanalytischen Annahme der Konstruktion von Subjekten, ihrer Identität und ihrer Körper mit Humor auf die Spitze getrieben. Nora Mansmann macht jedoch nicht nur auf die instabilen Grenzen einer intelligiblen Identität aufmerksam, indem sie eine solche Identität als Effekt permanenten Zitierens ausstellt. Durch die Methode des Ausstellens der Produktionsprozesse, die auf die intelligible Identität eines Subjekts abzielen, und der Normen, die eine solche intelligible Position bedingen, werden diese entsprechend dem Anliegen Judith Butlers ${ }^{6}$ als historisch

6 »Die ästhetische Umsetzung eines verletzenden Ausdrucks kann den Ausdruck sowohl verwenden als auch erwähnen, d.h. sie kann ihn gebrauchen, um bestimmte Wirkungen hervorzurufen, aber sich auch zugleich auf die Verwendung beziehen und damit die Aufmerksamkeit darauf lenken, dass es sich um ein Zitat handelt; und damit diese Verwendung in einer Zitattradition situieren, um die Verwendung zu einem expliziten Beispiel von Diskursivität zu machen, das eher reflektiert als für ein gesichertes Verfahren der Normalsprache gehalten werden sollte. Eine ästhetische Umsetzung kann außerdem das Wort verwenden, es aber zugleich ausstellen, auf es zeigen, als ein Beispiel des Arbiträren der Sprache darstellen, das benutzt wird, um bestimmte Effekte zu erzeugen. In diesem Sinne kommt die se- 
veränderbar erkannt. Die der Sprache konstitutiv verbundenen Eigenschaften der Iterabilität und der permanenten Bedeutungsverschiebung werden zum Ausgangspunkt einer möglichen Subversion der Machtverhältnisse. Der erste Schritt ist mit der Entschleierung der Normen als kontingent getan.

Ohne verlässliche Subjektposition und definierbare Körpergrenzen, unfähig, die Innen- von der Außenwelt, das Ich vom Anderen zu unterscheiden, sind ihre Figuren untrennbar mit den permanenten Verschiebungen der in Diskursen präsenten Machtverhältnisse verbunden. Aus dieser Situation speisen sich einerseits der spezifische Humor der Texte und andererseits die Sehnsucht nach Bedeutung und Authentizität, die Mansmanns Figuren umtreibt und sie an einer fast schon romantischen Suche festhalten lässt:

»aber wenn ich doch mal zu mir komme in so einer situation so einer umgebung oder atmosphäre wie hier gewissermaßen kontemplativ dann fallen mir sachen ein zum beispiel stelle ich mir manchmal vor dass alles sich verändert dass die langweile plötzlich weg ist und das leben irgendwas bedeutet oder so« (Mansmann 2008: 11).

Auch das Theater von Volker Lösch arbeitet mit den Mitteln der Groteske. Im Zentrum steht bei ihm der Gesellschaftskörper, der - auf vergleichbare Weise wie der Individualkörper in den Texten Mansmanns Verwerfungen ausgesetzt ist, die er nicht zu überblicken vermag. Permanent ist er mit Grenzziehungen und Ausschlussverfahren befasst, deren diskursives Zustandekommen häufig verschleiert bleibt. In diesem Sinn kann man Volker Löschs Arbeiten als Versuche begreifen, diese Prozesse sichtbar zu machen: Durch die geografische und soziale Verortung des Personals, durch die Auseinandersetzung mit möglichst konkreten Segmenten der außertheatralischen >Wirklichkeit`, durch die offenkundige Montage von Diskursfragmenten und ihre groteske Zuspitzung werden gesellschaftliche Strukturen in ihrer Entstehung wahrnehmbar. Als solche können sie dann auch wieder zur Disposition gestellt werden.

mantische Leere des materialen Signifikanten in den Blick, als das leere Moment in der Sprache jedoch, das zum Ort einer semantisch überlaufenen Tradition und ihrer Wirkungen werden kann.« (Butler 2006: 158) 


\section{Literatur}

Behrendt, Eva/Burckhardt, Barbara/Wille, Franz (2008): Die Rechten sind nicht nur die Anderen. In: Theater heute, H. 2, S. 11-19.

Butler, Judith (2001): Psyche der Macht. Das Subjekt der Unterwerfung. Frankfurt am Main: Suhrkamp.

Butler, Judith (2006): Hass spricht. Zur Politik des Performativen. Frankfurt am Main: Suhrkamp.

Derrida, Jacques (1976): Signatur Ereignis Kontext. In: Ders.: Randgänge der Philosophie. Frankfurt am Main/Berlin/Wien: Ullstein, S. $124-155$.

Dürrenmatt, Friedrich (1998): Der Besuch der alten Dame. Zürich: Diogenes.

Foucault, Michel (1978): Die Machtverhältnisse durchziehen das Körperinnere. Ein Gespräch mit Lucette Finas. In: Ders.: Dispositive der Macht. Über Sexualität, Wissen und Wahrheit. Berlin: Merve, S. 104-117.

Foucault, Michel (2002): Die Wahrheit und die juristischen Formen. In: Defert, Daniel/Ewald, Francois (Hg.): Michel Foucault. Schriften in vier Bänden. Dits et Ecrits 2. Frankfurt am Main: Suhrkamp, S. 669792.

Foucault, Michel (2003): Vorlesung vom 14. Januar 1976. In: Defert, Daniel/Ewald, Francois (Hg.): Michel Foucault. Schriften in vier Bänden. Dits et Ecrits 3. Frankfurt am Main: Suhrkamp, S. 231-250.

Mansmann, Nora (2005): TERRORMUM. Frankfurt am Main: Verlag der Autoren.

Mansmann, Nora (2006): herr tod lädt nicht ein aber wir kommen trotzdem. Frankfurt am Main: Verlag der Autoren.

Mansmann, Nora (2008): zwei brüder drei augen. Frankfurt am Main: Verlag der Autoren.

Merck, Nikolaus/Pilz, Dirk (2005): Den Bogen spannen, bis die Sehne reißt. In: Theater der Zeit, H. 11, S. 16-19.

Rischbieter, Henning (1968): Editorial. In: Theater heute, H. 9, S. 1.

Schwiedrzik, Wolfgang/Stein, Peter (1968): Demokratie ist auch Aktion. In: Theater heute, H. 9, S. 2-3. 
Bereitgestellt von | Universitaetsbibliothek Basel

Angemeldet

Heruntergeladen am | 19.09.18 10:57 